\title{
Identifying Critical Success Factors through Interactive Management
}

\author{
Nien-Tsu $\operatorname{Tuan}^{1 *}$ \\ ${ }^{1}$ Department of Construction Economics and M anagement, University of Cape Town, South A frica
}

\begin{abstract}
Since its inception, the C ritical Success F actor (CSF) concept has been increasingly adopted by industries to achieve business goals. How ever, the conventional approaches used for identifying critical success factors are underpinned by the mechanism paradigm. The mechanism paradigm doesn't take the interlaced relationships between system elements into account. $\mathrm{N}$ either does it promote interaction between the relevant stakeholders. This paper proposes a systemic approach called Interactive M anagement (IM) to complement conventional ideas in determining the critical success factors. The Interactive $M$ anagement process embraces the relevant stakeholders to collectively identify the critical success factors through four phases: generating critical success factors, clarifying the generated critical success factors, structuring a systemic digraph showing the interlaced relationships between the critical success factors, and identifying the real critical success factors in the systemic digraph. A n example is provided to demonstrate how the Interactive $M$ anagement methodology can be used to identify the real critical success factors.
\end{abstract}

Keywords: critical success factors, interactive management, systemic approaches

\section{Introduction}

Since its inception, the Critical Success Factor (CSF) idea has been gaining ground in the identification of competitive strategy for organizations. It has been used for establishing business strategies in various industries, e.g. R\&D projects [1], automobile industry [2], and mobile learning [3]. However, the frequently used techniques for exploring CSFs are dominated by the thinking that sees human as subjects, treats systems elements as independent parts, or narrows down the scope of the investigation to a desirable level. These techniques can be exemplified by means of interviews, Likert Scale questionnaire surveys, or statistical correlation analysis. Different from the commonly adopted techniques, this paper uses an example to demonstrate how the Interactive Management methodology [4] can be employed to identify and prioritize CSFs. Interactive Management (IM) is a systemic approach echoing soft systems thinking. It enhances learning and interchanging perceptions

* Corresponding author: nien-tsu.tuan@uct.ac.za 
among the relevant stakeholders through a disciplined process: generation of ideas, clarification of generated ideas, structuring the generated ideas, and interpretation of structured ideas. The output of the IM process is a digraph depicting the relationship between the system's elements. The following discussion will demonstrate how to use IM to structure a CSF model. Prior to the demonstration, this paper explains the concept of CSF and the techniques frequently used to identify the CSFs.

\section{Critical Success Factors}

The CSF concept was first put forward by Daniel [5]. It means the key areas that must be exceptionally well done for an organization to be successful. This idea was then popularized by Rockart [6], using CSF method to identify the key areas where things must be done right for different companies, such as Microwave Associates, an oil company, and a store-furnishing manufacturer. In some ways, one of the objectives of the CSF method is to reduce the variety of decision making impinging on top executives/general managers. They are frequently furnished with an enormous amount of unrelated information. The CSF method aims to provide executives with critical information derived from the identified critical areas requiring attention. With the attenuated variety of information derived from the critical areas, the executives can manage their organizations in an efficient and effective fashion.

Since its emergence, the CSF concept has been used in different areas. For example, Pinto and Slevin [7] used it to identify the critical factors in successful project implementation. They identified ten factors promoting project success. The ten factors range from the factor sweeping across all activities in various projects, like the factor 'communication', to the factor focusing on a specific project stage, such as the factor 'project schedule/plan'. Mahmood et al. [8] explored the critical success factors in research projects at universities in Pakistan. They concluded eleven critical success factors for research projects, such as project safety, team members' relationship, and transparency. Apparently, the eleven critical success factors identified by them for research projects are not identical to the ten critical success factors of generic projects identified by Pinto and Slevin [6]. In addition to exploring the project management arena, the CSF idea leaves visible traces in other issues as well. Cochrane [3] investigated the critical success factors in transforming pedagogy towards mobile learning. Luthra et al. [2] probed into the critical success factors for achieving sustainability in the automobile industry. Sahney [9] studied the critical success factors for E-business. For Sahney, the critical success factors refer to the design characteristics of meeting customers' requirements. The CSF concept has profoundly influenced the strategic planning in different industries.

There are some criticisms of the CSF idea. Cooper [10] summarizes various criticisms from the extant literature. For example, due to the limitation on short-term memory, managers might reduce CSFs to a manageable number. There may be other CSFs not taken into account. Focusing on a measurable factor is another problem: the focus on measurable factors could lead to the ignorance of unmeasurable 'soft' factors. Another concern is about the dynamic environment: the dynamic environment requires that the critical success factors identified for an organization should be frequently revisited to ensure their relevance to the organizational objectives. However, the criticisms didn't hamper the CSF idea that carves out its own line in different research domains through different techniques. The common approaches used to identify CSFs are briefly reviewed in the next part. 


\section{A Review of Commonly Used Approaches to the Identification of Critical Success Factors}

Using a literature review to generate a list of CSFs is a predominant method $[1,2,11,12]$. The collated CSFs from the literature may then be used to develop an instrument for surveys. The human subjects rate the CSFs predetermined by the researchers. The score received by each critical success, factor reveals how the predetermined CSFs are ranked by the subjects. The structured relationship between the CSFs appears to be 'more important'. This relationship is aimed at determining whether a critical success factor is more important than another critical success factor. But the spokesperson for CSF [6] urges researchers to note that the CSFs of each industry is characterized by the very nature of the industry itself. When zooming into organizational levels, the CSFs are amenable to change over time due to the change of an organization's situation. The idea of identifying invariant CSFs through literature to sweep across all industries and organizations is questionable.

Interviews are frequently used techniques in qualitative research. They are used for identifying CSFs as well $[6-8,13]$. In general, the interview procedures require researchers to transcribe the interview, code and categorize the data and discover the pattern of meaning from the coding and categorization. To defend that the coding and categorization are not biased towards a coder's subjectivity, Cohen's Kappa or other measures might be introduced into the procedures to defend the reliability of the data analysis. Overall, the findings should reflect trustworthiness, such as credibility, transferability, and dependability. In general, the output from the interview is a list of CSFs, without describing the relationship between the identified CSFs. Different from other interview outputs, Pinto and Slevin [7] argue that the CSFs in project implementation discovered by them display 'precedent to' relationship. For instance, the success factor 'project mission' is precedent to the success factor 'top management support'. In brief, an interview is a qualitative approach assuming that the summation of individual's perceptions is equal to the whole. The researchers' subjectivity influences the analysis of data to some degree. The problems of using an interview include lack of interaction among the human subjects and dismissing interrelationships between system elements. Even if member checking is used to justify the validity of findings, there is no guarantee that each participant understands why others uphold different views. The frequency count used to determine the most frequently mentioned factors by the participants doesn't promote consensus among them due to the lack of interaction.

Statistical analysis is another frequently used technique in a quantitative study. It has also been used to investigate the relationship between CSFs and other variables. For example, Dvira et al. [14] used the multivariate method to investigate the relationship between the CSFs of the defence projects and critical management variables. They mention that the multivariate method has proved to be useful in reducing the dimensionality of variables space. This idea is employed to identify the CSFs in their research as well. The CSFs were reduced from four success dimensions to two dimensions. This approach manifests the idea of 'narrowing down'. It is the idea of reducing the variables in question to a manageable level or matching a researcher's interest. However, the spokesperson of CSF adopted 'interview' to identify CSFs [6]. It implies that determining CSFs is a subjective business. Therefore, reducing the dimension of interest by the researcher alone doesn't deal with the subjectivity among the participants. The statistical analysis hints that reaching consensus among different views is subordinate to discovering invariant principles through statistics.

The reviewed methods that have been used for identifying CSFs are not exhaustive. There are other approaches used for identifying CSFs as well, such as Analytic Hierarchy Process [15]. In general, the reviewed methods are characterized by lack of interaction, reduction of problem space, seeing the identified CSFs invariant in time, or paying no heed of the interrelationships between system elements. Human is seen as subjects in most of the 
frequently used methods. Therefore the commonly adopted approaches do not enhance learning or dwell on the systemic relationship between system elements. Warfield devised Interactive Management (IM) methodology [16], a methodology aligned with soft systems thinking. IM can be used to structure the systemic relationship between CSFs in an interactive way. The next part explains how to use IM to identify CSFs and structure the interrelationships between the identified CSFs.

\section{Identifying CSFs through Interactive Management}

In its early development stage, IM was called Interpretive Structural Modelling [16]. Warfield and Cárdenas [4] elaborated Interpretive Structural Modelling and called the new methodology Interactive Management. IM has been used to study various problems. For example, Nthunya et al. [17] used IM to explore the major factors causing basic education problems in Lesotho. Tuan [18] applied IM to explore the aggravating relationship between the factors leading to the AIDS epidemic in South Africa. The earlier version of IM, i.e. Interpretive Structural Modelling, was also used to investigate CSFs [9, 19, 20]. However, these works need to be augmented by incorporating the vital features of IM into the inquiry. For example, given the democratic nature of IM [21], the facilitator of an IM workshop needs to justify that the constituency of the participants is representative. Furthermore, the interrelationship between the CSFs to be structured is a major objective of IM inquiry. The interrelationship 'helps to achieve' is more adequate than 'leads to' because the 'helps to achieve' relationship can manifest the CSFs that have higher leverage to promote other CSFs. These structured interrelations can reveal the relative importance of the identified CSFs. The following discussion demonstrates how the major IM phases can be used to identify CSFs.

The first step of IM is to identify the relevant stakeholders who will be the participants of the IM workshop. The constituency of the participants should be justifiable, e.g. identifying the relevant stakeholders through the questions proposed by Manson and Mitroff [22]. The questions range from 'who is affected by the strategy' to 'who has an interest in it. It is noteworthy that the ideal number of participants in the IM workshop is between six to twelve participants [4]. The ideal number of participants shouldn't be seen as a sample size. The participants of an IM workshop can be seen as the representatives of a committee. It is not sensible to claim that the 'sample size' of a 'committee' is too small or too big. The major concern about the committee is whether it is representative and knowledgeable. Assume that a company intends to identify the CSFs for the business that they operate. The company has identified six participants who are willing to attend the IM workshop. Besides, the constituency of the six participants is legitimate.

The second step is to generate CSFs. Each participant carries out the silent generation of CSFs based on their own perceptions. The reason for generating CSFs silently is to avoid a power situation entering into the inquiry process. When each participant completes the generation of CSFs, the facilitator may start to collate the generated ideas for entering the next phase.

The third step is to clarify the generated CSFs. As people might use the same words to express different ideas or use different words to express the same idea, the clarification phase promotes the shared understanding of the generated ideas between the participants. This phase requires the participants to collectively review all of the generated ideas through the assistance of a facilitator. Therefore a properly equipped venue is needed for the group to collectively review the generated CSFs. In the process of reviewing the generated CSFs, the participants might decide to merge certain CSFs into one, delete certain CSFs, or rephrase the CSFs whose meanings are unclear. Assume that the participants of the scenario case have collectively generated and clarified eight CSFs, shown in Table 1. The list of CSFs in Table 
1 is only used for demonstration purpose. In the real world, more CSFs could be generated for modelling.

Table 1. Example of Identified Critical Success Factors

\begin{tabular}{cc}
\hline Serial Number & Critical Success Factors \\
\hline 1 & a skilful team \\
2 & resources / financial support \\
3 & top management support \\
4 & recognition of project team \\
5 & absence of red tape \\
6 & sharing high-quality information \\
7 & customer support \\
8 & establishment of clear measurement of success \\
\hline
\end{tabular}

The third step is to structure a model/digraph showing the interrelationship between the identified CSFs. The digraph is structured by the group through answering a series of contextual questions examining whether the relationship exists between a pair of systems' elements. To determine the relative importance of CSFs, the contextual question 'Does the achievement of critical success factor A significantly helps to achieve the critical success factor B' may be used to build the model. The wording 'significantly' can help the group to avoid linking two CSFs when the intensity of the relationship between them is weak. The 'achievement of A helps to achieve B' means that attaining factor A has the power to promote factor B. If success factor A has the power to promote success factor B, success factor A should be given focus from a strategic point of view. It is noteworthy that the relationship in the model must be transitive. The 'helps to achieve' relation is transitive. If success factor A helps to achieve success factor B and success factor B helps to achieve success factor C, then it is plausible to infer that success factor A helps to achieve success factor $\mathrm{C}$. The use of 'lead to' in the contextual question is questionable. The phrase 'lead to' has been used for both pleasant and unpleasant situations. This phrase might give rise to confusion in the modelling process because, in addition to the 'help to achieve' relationship, 'inhibiting' relationship might exist between the CSFs as well. Therefore, it is not adequate to use 'lead to' for the contextual question due to the possibility of causing semantic confusion.

When the group answer the series of contextual questions, a binary matrix is gradually completed. If the group decides that the relationship exists between the two posed elements, ' 1 ' is entered into the cell associated with the two elements in the binary matrix. If not, ' 0 ' is entered into the cell. Once the binary matrix is completed, a digraph can be extracted. Warfield [16] explains the process of extracting the digraph. But it is not in the scope of this paper. Currently, there is software capable of carrying out the modelling process and extracting the digraph, such as Concept Star. The software provides the options for modelling loops in the system. Assume that the group carried out extensive debate and answered all questions required to complete the binary matrix. The digraph extracted from the binary matrix is shown in Figure 1. 


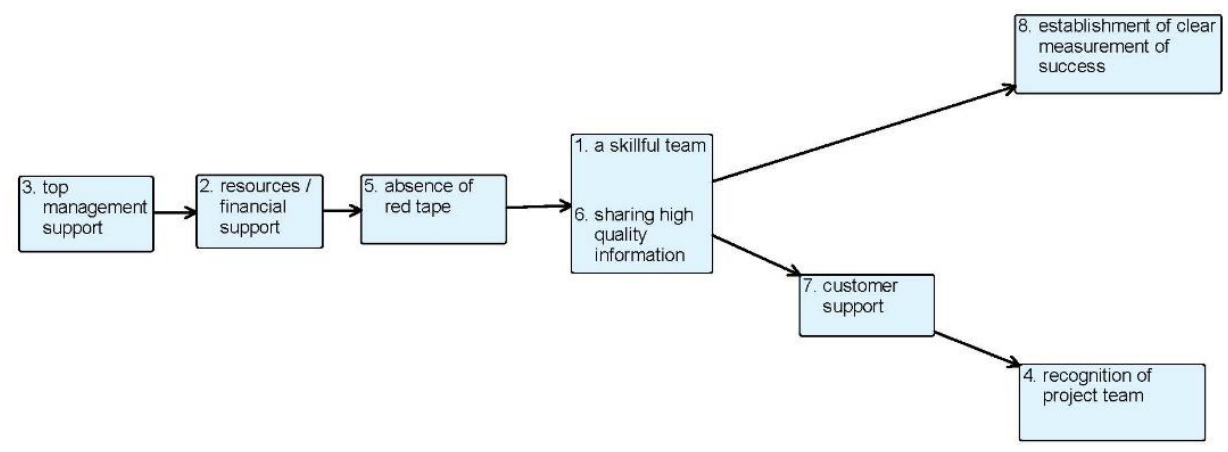

Fig. 1. An Example of 'Help to Achieve' Relationship between Critical Success Factors

The fourth step is to interpret the model built by the group. The facilitator of an IM workshop should be knowledgeable about IM and be able to carry out the interpretation of the structured model. In Figure 1, all of the arrows mean 'significantly helps to achieve'. For example, the leftmost element is critical success factor 3 'top management support'. The second stage element is critical success factor 2 'resources/financial support'. The arrow linking the two elements means that the achievement of critical success factor 'top management support' significantly helps to achieve the critical success 'resources/financial support'. The figure shows that the leftmost element 'top management support' can generate the greatest leverage because it significantly helps to achieve all of the critical success factors lying to its right. Therefore, critical success factor 3 'top management support' should be given priority from a strategic point of view. The second important element is the critical success factor lying in the second stage: critical success factor 2 'resources / financial support'. This factor has significant power to achieve other critical success factors. As for the two rightmost elements (establishment of clear measurement of success $\&$ recognition of project team), they have no power to achieve other critical success factors. Therefore, they shouldn't be given priority. Furthermore, Figure 1 shows a loop existing between two CSFs. The loop exists in the fourth stage box comprising two system elements: factor 1 (a skilful team) and factor 6 (sharing high-quality information). This box means that the achievement of 'a skilful team' significantly helps to achieve 'sharing high-quality information' and the achievement of 'sharing high-quality information' significantly helps to achieve 'a skilful team'. The loop reveals the systemic nature of CSFs which is a component leading to complexity.

The model structured in Figure 1 is not aimed at establishing universal laws. The IM methodology emphasizes learning and consensus. Through the IM process, the participants learn why others view the problems in different ways. The interactive process of building a model enhances the consensus among the relevant stakeholders. Because the environment and perceptions might change over time, the built model serves as the starting point for iterative learning.

\section{Conclusions}

This paper demonstrates how IM can be used for identifying/prioritizing CSFs. The output of the IM process is a digraph depicting the holistic relationship between the identified CSFs. This output is not visible in the methods frequently used for identifying CSFs in the surveyed literature, such as interview, questionnaire survey, and statistical analysis. In order to pick out the CSFs that can generate the greatest leverage, this paper proposes to use the contextual 
relationship 'help to achieve' for modelling. The model constructed in Figure 1 demonstrates the ripple effect of 'help to achieve' between the identified CSFs. In addition to the 'help to achieve' relationship, the foregoing paragraphs argue that 'inhibiting' relationship might exist between the CSFs as well. For example, assuming 'firmly adhering to the scope of work' and 'customer support' are both critical success factors. The success factor 'firmly adhering to the scope of work' might inhibit 'customer support'. 'Firmly adhering to the scope of work' implies the rejection of all customers' requests for the change in the scope of work, including minor variations. It is unlikely to obtain support from customers if their requests for minor changes are completely rejected. The IM can be further used to structure the 'inhibiting' relationship between CSFs to broaden the spectrum of appreciating CSFs. However, IM is a methodology anchored in soft systems thinking. Its inquiry process is to pursue learning and consensus among the stakeholders, rather than eternal laws.

\section{References}

1. N. DS, S. Thomas, Success factors of public funded $R \& D$ projects, Current science 108(3):357-363 (2015)

2. S. Luthra, D. Garg, A. Haleem, Critical success factors of green supply chain management for achieving sustainability in Indian automobile industry, Production planning \& control 26(5):339-362 (2015)

3. T.D. Cochrane, Critical success factors for transforming pedagogy with mobile web 2.0, British journal of educational technology 45(1):65-82 (2014)

4. J.N. Warfield, A.R. Cárdenas, A handbook of interactive management. A mes: Iowa State University Press (1994)

5. D.R. Daniel, M anagement information crisis. Havard business review 39(5):111-121 (1961)

6. J.F. Rockart, Chief executives define their own data needs, Havard business review 57(2):81-93 (1979)

7. J.K. Pinto, D.P. Slevin, Critical factors in successful project implementation, IEEE transactions on engineering management 34(1):22-27 (1987)

8. A. M ahmood, F. A sghar, B. N aoreen, "Success factors on research projects at university" an exploratory study, Procedia social and behavioral sciences 116:2779-2783 (2014)

9. S. Sahney, Critical success factors in online retail - an application of quality function deployment and interpretive structural modeling, International journal of business information 3(1):144-163 (2008)

10. V.A. Cooper, The critical success factors method: a review and practical example, International conference on information resource (2008)

11. D. Baccarini, Critical success factors for projects, ANZAM Conference, Western A ustralia, (2003)

12. A. Shenhar, O. Levy, D. Dvir, Mapping the dimensions of project success, Project management journal 28(2):5-13 (1997)

13. M.H. Shah, F.A. Siddiqui, Organisational critical success factors in adoption of e-banking at the Woolwich bank, International journal of information management 26(6):442-456 (2006)

14. D. Dvira, S. Lipovetskya, A. Shenharb, A. Tishlerc, In search of project classification: a non-universal approach to project success factors, Research policy 27(9):915-935 (1998)

15. D.K.H. Chua, Y.C. Kog, P.K. Loh, Critical success factors for different project objectives, Journal of construction engineering and management 125(3):142-150 (1999)

16. J.N. W arfield, Social systems: planning, policy and complexity, N ew Y ork: W iley (1976) 
17. M. N thunya, N.T. Tuan, C. Shaw, I. Jay, A systemic exploration of Lesotho $\odot$ basic education through Interactive Management, Systemic practice and action research 30(3):257-276 (2017)

18. N.T. Tuan, A systemic inquiry into the AIDS epidemic in the Western Cape of South Africa through Interactive Management, Systemic practice and action research 31(4):421-435 (2018)

19. S. Tripathy, S. Sahu, P.K. Ray, Interpretive structural modelling for critical success factors of $R \& D$ performance in Indian manufacturing firms, Journal of modelling in management $8(2): 212-240$ (2013)

20. D.K. Y adav, A . B arve, A nalysis of critical success factors of humanitarian supply chain: an application of Interpretive Structural M odeling, International journal of disaster risk reduction 12:213-225 (2015)

21. G. C. A lexander, Interactive management: an emancipatory methodology, Systemic practice and action research 15(2):111-122 (2002)

22. M.C. Jackson, Systems thinking: creative holism for managers, Chichester: W iley (2003) 